\title{
Relaxation channels and transfer of energy stored by pre-irradiated rare gas solids
}

\author{
E.V. Savchenko, I.V. Khyzhniy, and S.A. Uyutnov \\ B. Verkin Institute for Low Temperature Physics and Engineering of the National Academy of Sciences of Ukraine \\ 47 Lenin Ave., Kharkov 61103, Ukraine \\ E-mail: elena.savchenko@gmail.com
}

G.B. Gumenchuk, A.N. Ponomaryov, and V.E. Bondybey

Lehrstuhl für Physikalische Chemie II TU München, Lichtenbergstraße 4, Garching 85747, Germany

Received November 20, 2008

\begin{abstract}
The processes of energy relaxation in rare gas solids pre-irradiated with an electron beam are discussed. We studied the emission of exoelectrons and photons from RGS. Investigations were performed by a set of activation spectroscopy methods applied simultaneously to each sample. Photon-stimulated exoelectron emission from solid $\mathrm{Ne}$ was observed for the first time.
\end{abstract}

PACS: $72.20 . \mathrm{Jv}$ Charge carriers: generation, recombination, lifetime, and trapping;

78.60.Kn Thermoluminescence;

79.75. $+\mathrm{g}$ Exoelectron emission;

78.55.-m Photoluminescence, properties and materials;

78.60.-b Other luminescence and radiative recombination.

Keywords: relaxation processes, energy transfer, activation spectroscopy.

\section{Introduction}

The widest band gap insulators - rare gas solids (RGS) - are excellent model objects for studying the processes of energy and charge transfer in solids irradiated with some kind of ionizing radiation. Exposure of RGS to ionizing radiation results in generation of electron-hole pairs, followed by a sequence of relaxation processes involving a variety of radiation induced defects defects of structure, self-trapped/trapped holes, electrons trapped at some lattice imperfections or by impurities with positive electron affinity. These centers are capable to store a part of the energy absorbed during excitation for quite a long time at low temperatures. To understand the radiation effects one has to study different channels of relaxation processes. Investigations on this subject have been spread to different classes of materials [1-4].

Up to very recently there were not so many publications on the activation spectroscopy study of RGS. In Refs. 5-7 the authors applied thermally stimulated luminescence (TSL) method; in Ref. 8 thermally stimulated current spectroscopy was used. In the case of doped RGS or RGS containing some impurity centers TSL may originate both from recombination of charged particles and from recombination of neutral atoms. Recent studies of relaxation in pre-irradiated rare gas solids revealed new radiative mechanism of energy transfer from atomic to electronic subsystem [9]. To study the whole set of relaxation channels and their interconnection there is a clear need to combine optical and current spectroscopy methods, e.g., TSL and thermally stimulated exoelectron emission (TSEE). Such a combination of activation spectroscopy methods is a highly efficient tool for studying channels of energy relaxation in pre-irradiated solids and for probing defect energy levels in the energy gap. We developed a low-temperature modification of correlation spectroscopy - synchronous measurements of TSL, TSEE and desorption of neutral atoms [10] from pre-irradiated RGS. In our previous experiments TSEE was detected from solid Ar [11] and $\mathrm{Ne}$ [12,13]. Thermally stimulated techniques were complemented by photon-stimulated luminescence (PSL) [14] and photon-stimulated exoelectron emission (PSEE) methods [15]. 
In this article we present the brief review of our activation spectroscopy investigations of pre-irradiated RGS. New results on TSL and TSEE from solid Ar and Xe are presented as well as PSEE from solid Ne, Ar and Xe.

\section{Experiment}

For preparation of Ne, Ar and Xe cryocrystals high purity gases (99.9996\%) were used. Before the experiment the gas inlet system was pumped and degassed by heating under pumping up to pressure $10^{-8}$ mbar. The samples were condensed from the gas phase under isobaric conditions $\left(P=10^{-7}\right.$ bar) on a metal substrate cooled by a closed-cycle 2-stage Leybold RGD 580 cryostat. A deposition rate was kept at about $10^{-2} \mu \mathrm{m} / \mathrm{s}$. A typical sample thickness was $100 \mu \mathrm{m}$.

The samples were irradiated with an electron beam. We used electrons of $500 \mathrm{eV}$ energy and the current density of $30 \mathrm{~A} / \mathrm{cm}^{-2}$. The irradiation of the samples was performed at low temperature $(6 \mathrm{~K})$ in order to exclude the conventional thermal mechanism of defect creation and to avoid the annealing of the radiation-induced defects. On completion of the irradiation the spectrum of TSL and TSEE yield were recorded using a linear heating. The heating rate was $3,2 \mathrm{~K} / \mathrm{min}$. The yield of TSL was measured with photon multiplying tube sensitized to VUV light.

In TSEE experiments the emission of electrons from pre-irradiated samples was detected with an Au-coated Faraday plate kept at a small positive potential $+9 \mathrm{~V}$. The current from the Faraday plate was amplified by a FEMTO DLPCA 100 current amplifier. The signal was reversed in polarity by an inverter and digitized in a PC.

For experiments on photon stimulated exoelectron emission we used a Coherent 899-05 dye laser pumped with Ar-ion laser operating in a continuous mode. While RGS samples are transparent for visible and infrared light their heating under laser beam can be caused only by heat transfer from the substrate. Its temperature monitored during experiment did not exceed $0,5 \mathrm{~K}$.

\section{Discussion}

Figure 1 demonstrates the total yield of solid $\mathrm{Ne}$ thermoluminescence in comparison with the dependence of exoelectron emission current on temperature. The main feature on the TSEE curve is the peak at $10.5 \mathrm{~K}$ with low-temperature shoulder at $9.5 \mathrm{~K}$. The peak about $13 \mathrm{~K}$ is caused by sublimation of Ne sample. The TSL glow curve also has a broad maximum at $10.5 \mathrm{~K}$.

The correlation of $10.5 \mathrm{~K}$ peaks in the TSL and TSEE yields points to the common primary process for these phenomena - release of electrons from the same kind of traps. Note that in all RGS free electrons are highly mobile and are responsible for charge transport. In case of

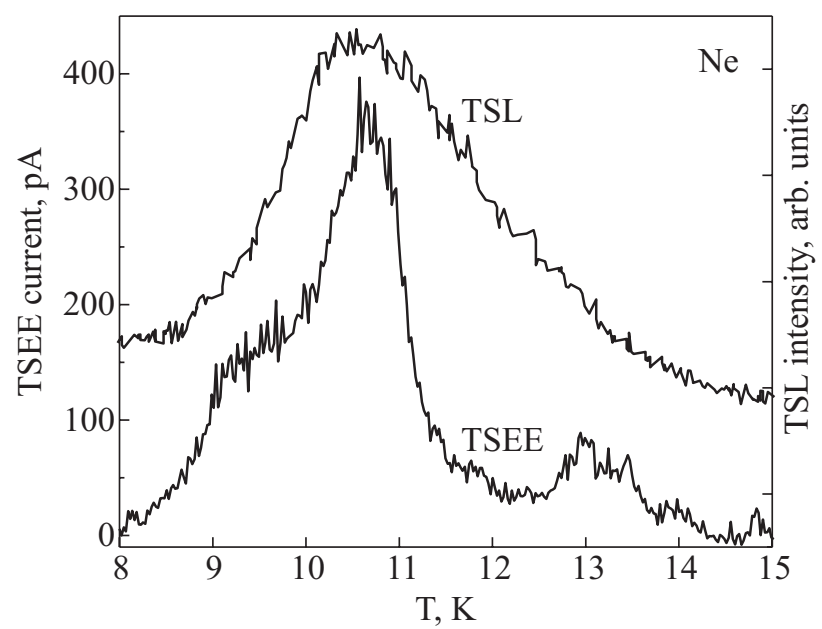

Fig. 1. Correlation of the TSL total yield and TSEE current from solid $\mathrm{Ne}$.

solid $\mathrm{Ne}$ (as well as for solid Ar) there is no energy barrier for electrons to escape from the sample to the vacuum because of negative electron affinity $(-1.3 \mathrm{eV}$ for $\mathrm{Ne})$ [4].

The value $10.5 \mathrm{~K}$ is the characteristic temperature for electronically induced structural defects annihilation in solid Ne. The atomic configuration of these point defects considered theoretically in Ref. 16 is the second-neighbor Frenkel pairs in the vicinity of the atomic-type selftrapped exciton in the Ne lattice. Note that because of negative electron affinity of solid $\mathrm{Ne}$ the vacancies in the Frenkel pairs serve as traps for electrons. The authors calculated the barrier height $(E=20 \mathrm{meV})$ corresponding to the formation of these defects. This value was estimated from the descending part of the TSEE curve in Fig. 1 [12] using the method suggested by [17] and using isothermal decay and different heating rates methods [13]. The estimations from the experimental results were found to be in a good agreement with the theory providing support for the suggested model.

In Fig. 2 a similar correlation of the TSL and TSEE from the crystalline $\mathrm{Xe}$ is shown. The TSL and TSEE peaks correlate in their position that indicates identity of the first step for each peak appearance: release of electrons from the traps of the same activation energy. However there is a competition between TSL and TSEE processes because free electrons have two different possibilities - to recombine with positively charged center or to escape from the surface of the crystal. It was found that at low temperatures where the shallowest traps situated (predominantly in the surface range) TSEE predominates. As the temperature is increased, the probability of recombination reactions increases as evidenced by rise of the intensity of recombination emission in comparison with the TSEE yield in temperature range $20-40 \mathrm{~K}$. Taking into account the bulk origin of the peaks in this range it suggests close positions of positively charged centers $\left(\mathrm{Xe}_{2}^{+}\right)$and trapped electrons. At the further temperature increase to 


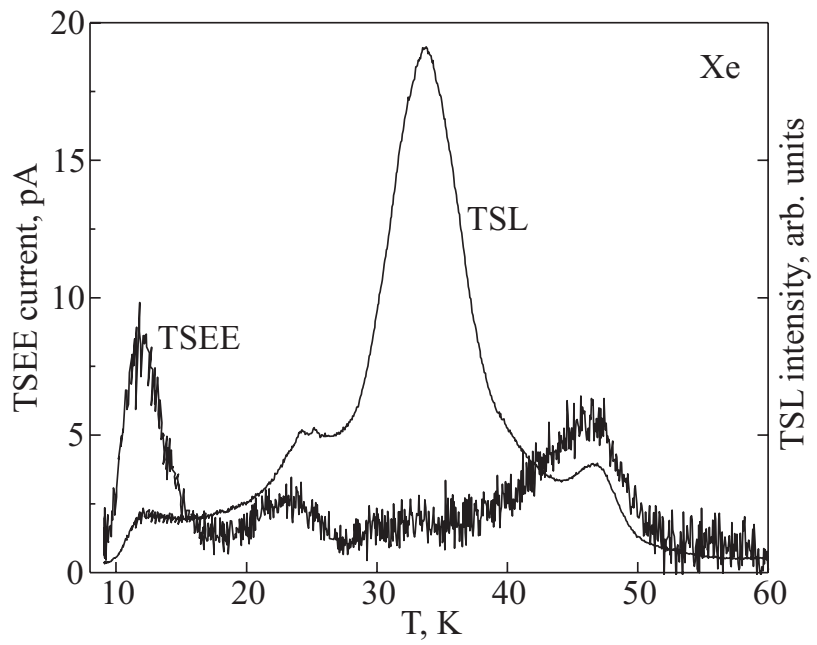

Fig. 2. Correlation of the TSL total yield and TSEE current from solid Xe.

$45 \mathrm{~K}$ charge recombination and exoelectron emission became equiprobable indicating an enlargement of the charge separation. Point structural defects in solid Xe can be traps for electrons as follows from observation of thermally stimulated intrinsic recombination luminescence stemmed from the reaction [18]

$$
\mathrm{Xe}_{2}^{+}+e \rightarrow \mathrm{Xe}_{2}^{*} \rightarrow \mathrm{Xe}+\mathrm{Xe}+h \mathrm{v}
$$

In view of positive electron affinity of solid Xe only such defects as interstitial atoms can catch electrons but not vacancies, pores etc. An effect of the number of structural defects in the sample of solid Xe on TSL and TSEE yields was shown in Ref. 19.

Taking into account the positive electron affinity of solid Xe $0.5 \mathrm{eV}$ [4] one can deduce that the peak of TSEE should be shifted to the high-temperature side in comparison with the corresponding TSL peak. We did not observe any shift. This could be caused by the space charge of the sample accumulated during irradiation with electrons. But this supposition needs further experimental verification.

The yields of TSL and TSEE from the preliminarily annealed sample of solid Ar are shown in Fig. 3. The common origin of these phenomena is obvious from the correlation of their intensities rises and peak positions. The TSL glow curve consists of the overlapping peaks of different origin at 12,17 and $23 \mathrm{~K}$. The first peak in the TSL correlates with $12 \mathrm{~K}$ TSEE peak and stems from the defects in subsurface layer. The second TSL maximum is related to the radiation-induced defects and appears after the corresponding TSEE peak.

The maximum of TSL at $23 \mathrm{~K}$ is caused by the presence of oxygen atoms in the sample, which come from the residual gases in the vacuum chamber. For the oxygen-doped solid Ar this maximum predominates in the glow curve. Chemiluminescence from the oxygen-con-

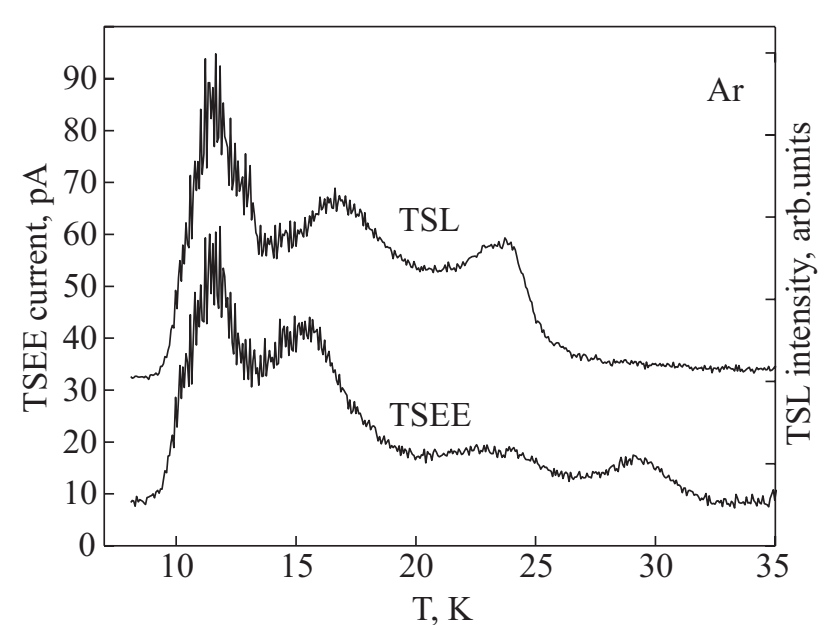

Fig. 3. Correlation of the TSL total yield and TSEE current from solid Ar.

taining RGS is quite common and was extensively studied in Ref. 20. The origin of the $23 \mathrm{~K}$ peak in TSL and TSEE was discussed in Ref. 21. It is caused by the recombination of neutral oxygen atoms due to increase of their mobility under heating and the following formation of the excited molecule, which emits the photon under transition to the ground state. This visible range emission releases electrons from the traps and triggers the relaxation cascades including the intrinsic recombination emission and exoelectron emission. Thus chemiluminescence affects the relaxation processes in the pre-irradiated RGS. Effect of visible range photons on pre-irradiated RGS is demonstrated in Fig. 4.

All PSEE experiments were performed with the same power $\left(35 \mathrm{~mW} / \mathrm{cm}^{2}\right)$ and the same wavelength $(510 \mathrm{~nm})$

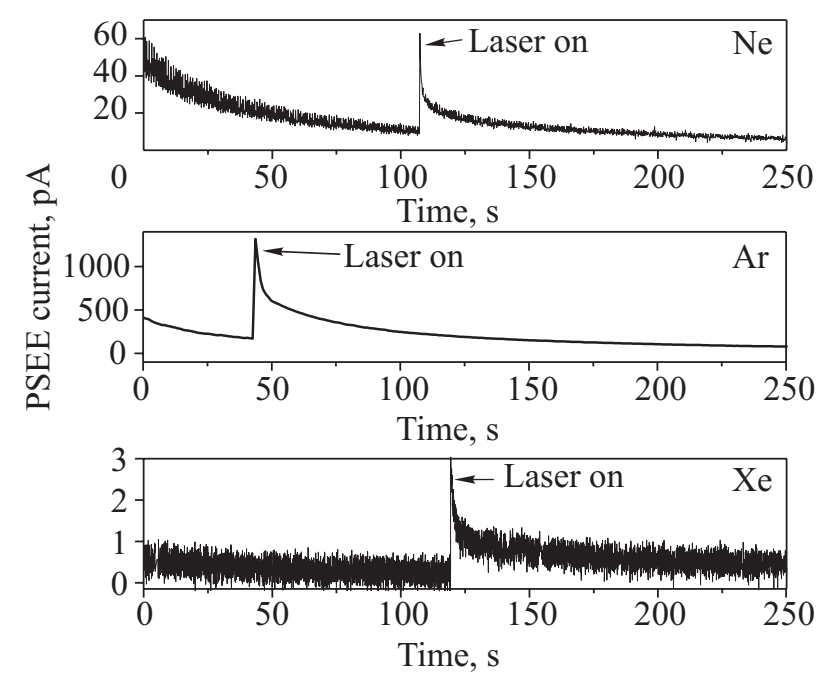

Fig. 4. The photon-stimulated exoelectron emission from solid $\mathrm{Ne}, \mathrm{Ar}$ and $\mathrm{Xe}$ at $T=6 \mathrm{~K}$. The irradiating electron beam was switched off at $t=0$. The samples were exposed to the laser beam when afteremission decayed and took on a constant value. 
of the laser beam. In all RGS samples we observed the decaying emission of electrons after switching off the irradiating electron beam. The so-called afteremission is caused by the presence of metastable $\mathrm{N}$ atoms in the lattice from the residual gases in the chamber. These centers are formed during the irradiation of the sample with electrons and exhibit long-lifetime green afterglow at $\lambda=$ $=520 \mathrm{~nm}$ after switching off the irradiating beam. This internal source of photons causes the phenomenon of «afteremission».

When «afteremission» was complete we switched on the laser focused to the surface of the sample and a strong increase of exoelectron emission current was observed. The highest value of PSEE current was detected from solid Ar while the lowest - from solid Xe. Due to wide conduction band in RGS the visible light can release electrons both from shallow and deep traps. The current decayed in time exponentially because of depopulation of the electron traps under the influence of the laser light. The initial parts of the decay curves can be fitted by the first-order exponential functions with characteristic decay time $\tau=(25 \pm 4) \mathrm{s}$ for solid $\mathrm{Ne}, \tau=(26 \pm 4) \mathrm{s}$ for solid $\mathrm{Ar}$ and $\tau=(15 \pm 4) \mathrm{s}$ for solid Xe. These PSEE experiments confirmed the idea that radiative transitions of metastable centers or chemiluminescence of neutral dopant atoms in the pre-irradiated RGS can strongly affect the processes of charge and energy transfer.

\section{Summary}

We applied activation spectroscopy methods - thermally stimulated luminescence in combination with thermally- and photon-stimulated exoelectron emission measurements to probe relaxation processes in solid $\mathrm{Ne}$, Ar and Xe pre-irradiated with an electron beam. Three relaxation channels were revealed: (i) recombination of charged particles and (ii) recombination of neutral ones both followed by photon emission in VUV and visible range correspondingly and (iii) emission of exoelectrons. A correlation in the positions of TSL and TSEE peaks pointed to the common primary process - electron detrapping from the same kind of traps. A competition between branching relaxation channels (i) and (iii) was demonstrated. Recombination of neutral atoms (ii) is considered to play the role of stimulating factor triggering (i) and (iii) channels. The relaxation scenario is shown to operate in both cases - RGS with negative and positive electron affinities.

The authors thank Profs K.S. Song, G. Zimmerer and M. Kirm for valuable discussions. I.V. Khyzhniy, E.V. Savchenko, and S.A. Uyutnov would like to thank DFG for the travel grant.
1. N. Itoh and A.M. Stoneham, Material Modification by Electronic Excitations, University Press, Cambridge (2000).

2. M.A. Elango, Elementary Inelastic Radiation-Induced Processes, American Institute of Physics, New York (1991).

3. Ch.B. Lushchik and A.Ch. Lushchik, Decay of Electronic Excitations With Defect Formation In Solids, Nauka, Moscow (1989) (in Russian).

4. K.S. Song and R.T. Williams, Self-Trapped Excitons, Springer Series in Solid-State Science, v. 105, SpringerVerlag, Berlin (1996).

5. M. Kirm and H. Niedrais, J. Luminescence 60-61, 611 (1994).

6. M. Kink, R. Kink, V. Kisand, J. Maksimov, and M. Selg, Nucl. Instr. and Meth. B122, 668 (1997).

7. J. Becker, O.N. Grigorashchenko, A.N. Ogurtsov, M. Runne, E.V. Savchenko, and G. Zimmerer, J. Phys. D: Appl. Phys. 31, 749 (1998).

8. A. Schrimpf, C. Boekstiegel, H.-J. Stökmann, T. Bornemann, K. Ibbeken, J. Kraft, and B. Herkert, J. Phys.: Condens. Matter 8, 3677 (1996).

9. E.V. Savchenko and V.E. Bondybey, Phys. Status Solidi A202, 221 (2005).

10. E.V. Savchenko, I.V. Khyzhniy, G.B. Gumenchuk, A.N. Ponomaryov, M.K. Beyer, M. Frankowski, and V.E. Bondybey, Rad. Phys. Chem. 76, 577 (2007).

11. E.V. Savchenko, O.N. Grigorashchenko, A.N. Ogurtsov, V.V. Rudenkov, G.B. Gumenchuk, M. Lorenz, A. Lammers, and V.E. Bondybey, J. Low Temp. Phys. 122, 379 (2001).

12. O.N. Grigorashchenko, V.V. Rudenkov, I.V. Khyzhniy, E.V. Savchenko, M. Frankowski, A.M. Smith-Gicklhorn, M.K. Beyer, and V.E. Bondybey, Fiz. Nizk. Temp. 29, 1147 (2003) [Low Temp. Phys. 29, 876 (2003)].

13. M. Frankowski, E.V. Savchenko, A.M. Smith-Gicklhorn, O.N. Grigorashchenko, G.B. Gumenchuk, and V.E. Bondybey, J. Chem. Phys. 121, 1474 (2004).

14. G.B. Gumenchuk, M.A. Bludov, and A.G. Belov, Fiz. Nizk. Temp. 31, 237 (2005) [Low Temp. Phys. 31, 179 (2005)].

15. E.V. Savchenko, O.N. Grigorashchenko, A.N. Ogurtsov, V.V. Rudenkov, G.B. Gumenchuk, M. Lorenz, M. Frankowski, A.M. Smith-Gicklhorn, and V.E. Bondybey, Surface Science 507-510, 754 (2002).

16. K.S. Song and Chun-Rong Fu, J. Low Temp. Phys. 111, 645 (1998).

17. Ch.B. Lushchik, JETP 3, 390 (1956).

18. E.V. Savchenko, A.N. Ogurtsov, I.V. Khyzhniy, G. Stryganyuk, and G. Zimmerer, Phys. Chem. Chem. Phys. 7, 785 (2005).

19. I.V. Khyzhniy, O.N. Grigorashchenko, A.N. Ponomaryov, E.V. Savchenko, and V.E. Bondybey, Fiz. Nizk. Temp. 33, 701 (2007) [Low Temp. Phys. 33, 529 (2007)].

20. A.V. Danilychev and V.A. Apkarian, J. Chem. Phys. 99, 8617 (1993).

21. E.V. Savchenko, O.N. Grigorashchenko, G.B. Gumenchuk, A.G. Belov, E.M. Yurtaeva, M. Frankovski, A.M. Smith-Gicklhorn, and V.E. Bondybey, Surf. Science 528, 266 (2003). 